\title{
ONS economic and labour market publications
}

\section{ANNUAL}

Financial Statistics Explanatory Handbook

2007 edition. Palgrave Macmillan, ISBN 1-4039-9783-7. Price $f 45$

www.statistics.gov.uk/products/p4861.asp

Foreign Direct Investment (MA4)

2005 edition

www.statistics.gov.uk/products/p9614.asp

Input-Output analyses for the United Kingdom

2006 edition

www.statistics.gov.uk/products/p7640.asp

Research and development in UK businesses (MA14)

2005 edition

www.statistics.gov.uk/statbase/product.asp?vlnk=165

\section{Share Ownership}

2006 edition

www.statistics.gov.uk/products/p930.asp

United Kingdom Balance of Payments (Pink Book)

2007 edition. Palgrave Macmillan, ISBN 978-1-4039-9397-7. Price f49.50.

www.statistics.gov.uk/products/p1140.asp

United Kingdom National Accounts (Blue Book)

2007 edition. Palgrave Macmillan, ISBN 978-1-4039-9398-4. Price f49.50.

www.statistics.gov.uk/products/p1143.asp

\section{First releases}

- Annual survey of hours and earnings

- Business enterprise research and development

- Foreign direct investment

- Gross domestic expenditure on research and development

- Low pay estimates

- Regional gross value added

- Share ownership

- UK trade in services

- Work and worklessness among households

\section{QUARTERLY}

Consumer Trends

2007 quarter 1

www.statistics.gov.uk/products/p242.asp

United Kingdom Economic Accounts

2007 quarter 1. Palgrave Macmillan, ISBN 978-0-230-52618-1. Price f32.

www.statistics.gov.uk/products/p1904.asp

UK trade in goods analysed in terms of industry (MQ10)

2007 quarter 1

www.statistics.gov.uk/products/p731.asp

\section{First releases}

- Business investment

- Government deficit and debt under the Maastricht Treaty (six-monthly)

- GDP preliminary estimate

- International comparisons of productivity (six-monthly)

- Internet connectivity

- Investment by insurance companies, pension funds and trusts

- Productivity

- Profitability of UK companies

- Public sector employment

- UK Balance of Payments

- UK National Accounts

- UK output, income and expenditure

\section{MONTHLY}

Financial Statistics

July 2007. Palgrave Macmillan, ISBN 978-0-230-52590-0. Price 445.

www.statistics.gov.uk/products/p376.asp

Focus on Consumer Price Indices

June 2007

www.statistics.gov.uk/products/p867.asp

Monthly review of external trade statistics (MM24)

June 2007

www.statistics.gov.uk/products/p613.asp

Producer Price Indices (MM22)

June 2007

www.statistics.gov.uk/products/p2208.asp

First releases

- Consumer price Indices

- Index of services

- Index of production

- Labour market statistics

- Labour market statistics: regional

- Producer prices

- Public sector finances

- Retail sales index

- UK trade

\section{OTHER}

The ONS Productivity Handbook: a statistical overview and guide Palgrave Macmillan, ISBN 978-0-230-57301-7. Price f55.

www.statistics.gov.uk/about/data/guides/productivity/default. asp

\section{Labour Market Review}

2006 edition. Palgrave Macmillan, ISBN 1-4039-9735-7. Price f40.

www.statistics.gov.uk/products/p4315.asp

National Accounts Concepts, Sources and Methods

www.statistics.gov.uk/products/p1144.asp

Sector classification guide (MA23)

www.statistics.gov.uk/products/p7163.asp 